\title{
Mechanism of Calcite Co-Orientation in the Sea Urchin Tooth
}

\author{
Christopher E. Killian, ${ }^{\dagger, *}$ Rebecca A. Metzler, ${ }^{\dagger}$ Y. U. T. Gong, Ian C. Olson, ${ }^{\dagger}$ Joanna Aizenberg, ${ }^{\S}$ Yael \\ Politi, Fred H. Wilt, Andreas Scholl, Anthony Young, Andrew Doran, Martin Kunz, Nobumichi \\ Tamura, Susan N. Coppersmith, and P. U. P. A. Gilbert* ${ }^{\dagger}, \#$
}
Department of Physics, UniVersity of Wisconsin, Madison, Wisconsin 53706, Department of Molecular and Cell Biology, UniVersity of California, Berkeley, California 94720, School of Engineering and Applied Sciences, HarVard UniVersity, Cambridge, Massachusetts 02138, Department of Structural Biology, Weizmann Institute of Science, RehoVot 76100, Israel, and AdVanced Light Source, Lawrence Berkeley National Laboratory, Berkeley, California 94720

E-mail:pupa@physics.wisc.edu

\begin{abstract}
Sea urchin teeth are remarkable and complex calcite structures, continuously growing at the forming end and self-sharpening at the mature grinding tip. The calcite (CaCO3) crystals of tooth components, plates, fibers, and a high-Mg polycrystalline matrix, have highly co-oriented crystallographic axes. This ability to co-orient calcite in a mineralized structure is shared by all echinoderms. However, the physicochemical mechanism by which calcite crystals become co-oriented in echinoderms remains enigmatic. Here, we show differences in calcite $c$-axis orientations in the tooth of the purple sea urchin (Strongylocentrotus purpuratus), using high-resolution X-ray photoelectron emission spectromicroscopy (X-PEEM) and microbeam X-ray diffraction ( $\mu$ XRD). All plates share one crystal orientation, propagated through pillar bridges, while fibers and polycrystalline matrix share another orientation. Furthermore, in the forming end of the tooth, we observe that $\mathrm{CaCO} 3$ is present as amorphous calcium carbonate (ACC). We demonstrate that co-orientation of the nanoparticles in the polycrystalline matrix occurs via solid-state secondary nucleation, propagating out from the previously formed fibers and plates, into the amorphous precursor nanoparticles. Because amorphous precursors were observed in diverse biominerals, solid-state secondary nucleation is likely to be a general mechanism for the co-orientation of biomineral components in organisms from different phyla.
\end{abstract}

\section{Introduction}

Sea urchins use their teeth to bite food and to grind rock and other substrates on which they are situated. Some species of sea urchin use their teeth to burrow into the rock as a means of protection from predators ${ }^{1,2}$ and, in the case of intertidal species, including the $S$. purpuratus studied here, as protection from the surge of the pounding waves. The sea urchin teeth have interested naturalists since Aristotle first described them in his Historia Animalium ca. 343 BCE. Today, chemists, physicists, and materials and life scientists study sea urchin teeth for their complex architecture at the nano-and microscales, which has been highly refined by evolution. These intricate biological structures can inspire the nanofabrication of highly co-oriented synthetic materials. The five calcitic teeth of an adult sea urchin are continuously growing and are held in the jaw apparatus called Aristotle's lantern. The tooth is longitudinally elongated with a curved shape and a keel on its concave side that lends it a T-shape cross-section. ${ }^{4-9}$ The larger structural components of the tooth, plates and fibers, are formed by syncytia of odontoblasts in the plumula at the $\underset{4,6}{\text { proximal end of the tooth. }}{ }^{4,5}$ These components are assembled together and secreted onto the growing tooth. ${ }^{4,6}$ A polycrystalline matrix of $10-20 \mathrm{~nm}$ particles of Mg-rich calcite (40-45 $\mathrm{mol} \% \mathrm{Mg}$ ) subsequently fills the space between the plates and the fibers, effectively cementing all components together. All echinoderms align the calcite crystals of their mineralized structures in a highly cooriented fashion, making this interesting physical aspect a shared trait, a synapomorphy, of this phylum. 
Recently, Ma et al. ${ }^{12}$ reported that the teeth from the species Paracentrotus liVidus have two highly cooriented crystalline blocks oriented differently by a few degrees and that these crystalline blocks interdigitate near the grinding tip. They also reported that the calcite of all components in each block, the plates, the fibers, and the polycrystalline matrix, is highly cooriented from the nanometer to the centimeter scale. These observations raise important questions about how the tooth components co-orient one another during tooth formation. How do all plates share a single $c$-axis orientation, despite being physically separate? How does the polycrystalline matrix form and co-orient its crystal nanoparticles? Are the nanoparticles aggregating before or after crystallizing? In other words, is this the result of oriented attachment of crystalline nanoparticles, as first observed by Penn and Banfield in $\mathrm{TiO} 2{ }^{13}$ and $\mathrm{FeOOH},{ }^{14}$ and later in many other synthetic mesocrystals? ${ }^{15}$ Or, does an amorphous precursor phase form first and crystallinity propagate though it subsequently?

Ma et al. observed that the fibers, and possibly the plates, are initially composed of amorphous calcium carbonate (ACC).

The present study reveals for the first time that the forming sea urchin tooth contains not one but two amorphous precursor minerals. These precursor phases are identical to those reported by Politi et al. ${ }^{16}$ in $S$. purpuratus larval spicules. We combine scanning electron microscopy (SEM), X-ray photoelectron emission spectromicroscopy (X-PEEM), and microbeam X-ray diffraction ( $\mu \mathrm{XRD}$ ) measurements to obtain detailed spatially resolved information about the crystal orientations near the grinding distal end of the tooth. Our data support the hypothesis that crystallinity in the adult tooth components starts from a single small crystal and propagates through the fully formed anhydrous amorphous solid. In the study by Politi et al,. ${ }^{16}$ a high-resolution and clear pattern of crystallinity propagation could not be imaged. The three-dimensional shape of the larval spicule makes it very difficult to generate that sort of data with XPEEM. The sea urchin tooth used here does not have this impediment because one can examine polished flat surfaces.

\section{Results}

Calcite crystal overgrowth experiments, as devised by Aizenberg et al., ${ }^{18}$ confirm that the calcite of the $S$. pupuratus tooth is highly co-oriented on a macroscopic scale. The overgrowth results are described in Figures 1 and $\mathrm{S} 1$.

The orientation of calcium carbonate crystals, such as calcite, can be measured with high spatial resolution by X-PEEM, using polarization-dependent imaging contrast (PIC). ${ }^{19-21}$ A PIC map displays different carbonate crystal $c$-axis orientations as different gray levels. Despite their curved shape, the plates in the sea urchin tooth share one crystal $c$-axis orientation, while all fibers and polycrystalline matrix share another $c$-axis orientation, as shown in the PIC map of Figure 2A.

In cross sections, the plates appear to be physically separate from one another (Figure 2A) and interspersed with the polycrystalline matrix with no obvious topological connections. Each plate is assembled by a syncytium of odontoblast cells in the plumula at the forming end of the tooth. ${ }^{4,6}$ We looked for connections between the plates, which could explain how a single crystal orientation could propagate across plates. We did not find plate connections within teeth cross sections near the mature tip. Away from the tip, however, we found mineral pillar bridges connecting the plates at the outer edges. Along the tooth's convex side, there are two sets of bridges that connect the plates on either side of the tooth. The bridges in the S. purpuratus tooth are shown in Figures $2 \mathrm{~B}$ and $\mathrm{S} 2$. These bridges were previously described by Ma“rkel ${ }^{22,23}$ and others ${ }^{24,25}$ as anatomical links between the sea urchin teeth and the ligaments holding them in the Aristotle's lantern. Ma"rkel ${ }^{25}$ also noted that in the sea urchin these bridges were attached to small pillars that arise from the edge of primary plates at the growing end of tooth. Figure S2 
shows that the pillar bridges in S. purpuratus run along the entire length of the tooth and are broken off from the sides of the tooth closer to the distal grinding tip. We see similar pillar bridges in the teeth from other sea urchin species,

P. liVidus (Figure S3) and L. Variegatus (Figure S4). In Figure S5, we show that the bridges are clearly continuous with the crystalline plates. The mineral pillar bridges therefore provide continuity to the calcite crystals in the plates on each side of the tooth as they are formed at the growing end. It follows from this finding that two initial nucleation events at the forming end (one on either side of the tooth) are sufficient for all ensuing plates to be co-oriented.

Chemical analysis reveals that at the forming end of the sea urchin tooth, where plates and fibers are fully formed, but the polycrystalline matrix has not yet completely filled the space between them, three distinct $\mathrm{CaCO} 3$ mineral phases are present. These three different phases are identified by their calcium L-edge absorption spectra. The spectral line shape is unique for each mineral structure and identified by the relative intensities of the crystal field peaks. The corresponding spectra, collected by X-PEEM, are presented in Figure 3, where the crystal field peaks are indicated by arrows. Figure S6 shows the image of the forming tooth, and the pixels from which the spectra of Figure 3 were extracted. The three phases detected in the forming $S$. purpuratus tooth are identical to those observed by Politi et al. ${ }^{16}$ in the $S$. purpuratus larval spicules, also shown in Figure 3. It is important to note that the ACC spectra of Figure 3 were collected from solid portions of the forming sea urchin tooth, as shown in Figure S6.

The similarity of crystal phases found in larval spicules and forming adult teeth indicates that $S$. purpuratus harnesses the thermodynamics of type 1-to-type 2 and of type 2-to-type 3 transformations, and uses them in different parts of its body at different developmental stages.

Fortuitously we identified two unusual calcitic fibers in a specific area (Figures 4 and S7), with a crystal orientation that differs significantly from the rest of the surrounding tooth components. This uncommon case of fibers with stray crystal orientations provides compelling evidence that nanoparticles in the polycrystalline matrix are imparted with their crystal orientation by secondary nucleation. Specifically, the $C$-axis orientation (gray level) of the nanoparticles surrounding these two stray fibers in Figure 4A is identical to that of the fibers, but different from the rest of the surrounding tooth components. Thus the different calcite crystal orientation "spills out" of the fibers into the polycrystalline matrix immediately adjacent to the fibers. This secondary nucleation propagates out of the fibers despite the well-defined and abrupt fiber profile, which is clearly imaged in the Mg map of these same fibers displayed in Figure 4B. The SEM micrographs in Figure S7 show the same sharp edges of the fibers as does the X-PEEM Mg map. This observation provides further evidence that crystal orientation alone, and not elemental composition, propagates radially, starting from the fibers and outward into the immediately adjacent polycrystalline matrix nanoparticles.

Figure S8 shows the same two stray fibers at different polarization angles, confirming the result of Figure $4 \mathrm{~A}$.

We examined the same area of the tooth in Figures 4, S7, and S8 and analyzed the two stray fibers using $\mu \mathrm{XRD}$. These fibers' $c$-axes are oriented $14^{\circ}$ from that of the surrounding matrix, thus accounting for the relatively large grayscale contrast observed in the PIC maps of Figures 4A and S8. The plates in this region are only $3-4^{\circ}$ off from the surrounding matrix.

In other regions of the tooth, we also see crystallinity propagation via secondary nucleation initiating from the plates. Figure 5 shows $\mathrm{Ca}, \mathrm{Mg}$, and PIC maps at the center of the tooth's stone part. As already seen in Figures 2 and 4, the plates in Figure 5 have a different calcite crystal orientation from that of the matrix and the fibers, as indicated by their slightly different gray levels in PIC maps. In a few locations immediately outside the plate boundaries (arrows in Figure 5), the orientation of the matrix propagates out of the plates. Similarly as for the fibers above, the $\mathrm{Ca}$ and $\mathrm{Mg}$ maps show well-defined boundaries for the plates. Yet the crystal orientation is propagated through the plate boundaries into the adjacent polycrystalline matrix.

Finally, we examined a thin portion of polycrystalline matrix protruding at the tip of an S. purpuratus tooth using $\mu \mathrm{XRD}$ and found that indeed the diffraction pattern corresponds to that of $10 \mathrm{~nm}$ size $\mathrm{Mg}$-rich calcite particles, with their $c$-axes strongly co-oriented within $1^{\circ}$, as indicated by the average width of the 
reflections. From the indexed diffraction patterns, it is possible to derive the ratio of lattice parameters, $c / a$, which is

3.395 for the matrix, a value compatible with a Mg-rich calcite phase. In the same tooth, the edge of the single first plate protruding at the tip and overlapping the tip-most matrix shows a $c$-axis orientation, which is rotated by $3-4^{\circ}$ with respect to the matrix. In contrast to the matrix, the reflections from the plates are sharp, indicating good crystallinity with a ratio $c / a$ of 3.41 , a value close to the one of pure calcite (3.42). The grayscale contrast between plates and matrix observed in PIC maps (Figures 2A, 4A, and 5-PIC), therefore, may also arise from $3-4^{\circ} \mathrm{C}$-axis angles, although the crystal orientations were not measured in this stone part region by $\mu \mathrm{XRD}$, and one should not assume that the angular spreads are constant across the whole tooth. From $\mu \mathrm{XRD}$ of another $S$. purpuratus tooth, tripod-polished to a thin section, we see that within a single plate the co-orientation is much greater, with $c$-axis spread within $0.025^{\circ}$.

\section{Discussion}

The observation of pillar bridges connecting the plates on each side of the sea urchin tooth enables one to consider the mineral phase of all plates on each side of the tooth to be connected. Similarly, the polycrystalline matrix is connected and continuous across the tooth. These simple morphological observations explain the high degree of crystal $c$-axis coorientation observed in Figure 1. The mechanism by which crystallinity propagates through these connected structures, however, warrants further discussion. We observed it here in the polycrystalline matrix, but the same mechanism is most likely exploited during plate and fiber formation, because amorphous precursors were observed in these components in

S. purpuratus (data not shown) and in P. liVidus. On the basis of the observations presented here, we deduce that crystallinity propagates during tooth formation into the fully formed but still amorphous matrix. Conversion from ACC to crystalline calcite, with a long-range order and fixed crystal orientation, occurs via a mechanism of secondary nucleation. During secondary nucleation, an already-crystalline nanoparticle templates its crystal orientation to its neighboring amorphous nanoparticles. Secondary nucleation occurs upon contact. Because there is no change in mass or volume during crystallization, the individual $\mathrm{CaCO} 3$ groups in $\mathrm{ACC}$ do not need to move very far to be arranged in an ordered crystal, as in displacive transformations. ${ }^{26}$ Secondary nucleation can be viewed as analogous to classical crystal growth from solution, in which a growing crystal incorporates ions from solution, one at a time, upon contact with the already-formed crystal surface. The difference is that the ordered and disordered phases are both solid for secondary nucleation, and they were already in contact

with each other, as they were fully aggregated before crystallization.

The alternative mechanism of calcite co-orientation, ruled out here, is oriented attachment of nanoparticles, where nanoparticles are formed and fully crystallized before they aggregate. ${ }^{13}$ In this mechanism, there may or may not be amorphous precursor phases for individual separate nanoparticles. However, these nanoparticles must be completely crystalline before they aggregate by oriented attachment. In the sea urchin tooth, we clearly observe ACC spectra in aggregated solid portions of the forming sea urchin tooth. Any not-yet-aggregated ACC nanoparticles, if they exist, would dissolve away from the tooth during polishing. Because the final mature tooth, including the matrix, is fully crystalline, ACC nanoparticles must be interpreted as being precursors that crystallize after aggregation or precipitation. The presence of ACC therefore excludes oriented attachment as the mechanism for calcite co-orientation of the sea urchin tooth matrix. 
The secondary nucleation mechanism by which crystallinity propagates in the sea urchin tooth is different from that produced in the laboratory by Aizenberg et al. ${ }^{17}$ In that study, Aizenberg et al. generated a twodimensional ACC film deposited on a micropatterned substrate and converted it to calcite starting from a single nucleation site. This synthetic system is water-rich, and the crystallization mechanism involves dissolution and mass transport. Secondary nucleation in the sea urchin tooth is a solid-state transformation. Despite the differences, there are macroscopic similarities between the two final products: Aizenberg et al. produced a millimeter-scale two-dimensional co-oriented film of calcite. In the sea urchin tooth matrix, calcite coorientation extends across the entire tooth $(1.5 \mathrm{~cm})$ in three dimensions. Another important idea introduced by Aizenberg et al. is that microscopic organic structures within the single crystal provide stress relaxation, thus permitting the crystal to grow co-oriented to large sizes. ${ }^{17}$ It is possible that organic molecules serve a similar role in the sea urchin tooth, therefore explaining the co-orientation of the matrix up to the macroscopic scale.

Various authors have observed dissolution and reprecipitation ${ }^{27}$ and even reproduced large calcite crystals using a sea urchin spine as a mold. ${ }^{28}$ Our results in the sea urchin tooth are in agreement with the Sommerdijk group's results from synthetic calcite growth, templated by an organic Langmuir monolayer, and observed with time resolution during the transition. In both systems, ACC-to-calcite is a solid-state transformation, more than one type of ACC is involved, and no bonds are broken in the process.

\section{Conclusions}

We find that the aggregated $\mathrm{CaCO} 3$ mineral of the growing tooth contains ACC precursors. We also observe that the crystal orientation of the plates near the stone part of the S. purpuratus tooth differs by 3$4^{\circ}$ from the nearby fibers and matrix. We show that the co-orientation of the plates is maintained through pillar bridges and that polycrystalline matrix crystal coorientation within $1^{\circ}$ occurs via secondary nucleation, propagating out from the fibers, and to a limited extent from the plates, into the amorphous precursors. This mechanism explains the overall co-orientation of the calcite of the sea urchin tooth, despite the tooth's complex architecture. The matrix, which cements all other components together, plays a key role in both tooth function and performance, and the co-orientation mechanism described here may inspire new design strategies for the synthesis of self-assembled mechanical, electronic, or photo-voltaic nanocomponents.

Because ACC precursors were observed in the plates and the fibers of $S$. purpuratus (data not shown) and $P$. liVidus, ${ }^{8}$ crystallinity in these elements very likely propagates via secondary nucleation as well. The pillar bridges described here provide the route for propagation of the same orientation, via secondary nucleation, across subsequent plates.

Calcite co-orientation of skeletal elements is a shared trait across the entire phylum of echinoderms; therefore, the present results may apply to echinoderms in general. Studies in other echinoderms would corroborate if secondary nucleation provides the physicochemical basis for this echinoderm synapomorphy. Because amorphous precursors have been observed in many phyla, echinoderms, 30,31 mollusks, ${ }^{32,33}$ chordates, 34,35 the prevalence of secondary nucleation may be even broader.

Acknowledgment. We thank Lia Addadi and Steve Weiner for discussions and comments about the manuscript. We also thank Paul Voyles for tripod polishing. This work was supported by DOE award DEFG02-07ER15899, NSF award CHE-0613972, and UW-Vilas and Hamel Awards to PUPAG. The experiments were performed at the ALS, supported by DOE under contract DE-AC0205CH11231.

Supporting Information Available: Eight supporting figures and methods. This material is available free of charge via the Internet at http://pubs.acs.org. 
(1) Moore, H. B. In Physiology of Echinodermata; Boolootian, R. A., Ed.; John Wiley and Sons: New York, 1966.

(2) Nelson, B. V.; Vance, R. R. Mar. Biol. 1979, 51, 251-258.

(3) Otter, G. W. Biol. ReV. Cambridge Philos. Soc. 1932, 7, 89-107.

(4) Kniprath, E. Calcif. Tissue Res. 1974, 14, 211-228.

(5) Ma, Y. R.; Cohen, S. R.; Addadi, L.; Weiner, S. AdV. Mater. 2008, 20, 1555-1559.

(6) Märkel, K.; Titschack, H. Z. Morph. Tiere 1969, 64, 179-200.

(7) Wang, R. Z.; Addadi, L.; Weiner, S. Philos. Trans. R. Soc. London, Ser. B 1997, 352, 469-480.

(8) Ma, Y. R.; Weiner, S.; Addadi, L. AdV. Funct. Mater. 2007, 17, 2693- 2700.

(9) Wang, R. Z. J. Am. Ceram. Soc. 1998, 81, 1037-1040.

(10) Berman, A.; Hanson, J.; Leiserowitz, L.; Koetzle, T. F.; Weiner, S.; Addadi, L. Science 1993, 259, 776-779.

(11) Brusca, R. C.; Brusca, G. J. InVertebrates; Sinauer Associates: Sunderland, MA, 1990.

(12) Ma, Y. R.; Aichmayer, B.; Paris, O.; Fratzl, P.; Meibom, A.; Metzler,

R. A.; Politi, Y.; Addadi, L.; Gilbert, P. U. P. A.; Weiner, S. Proc. Natl. Acad. Sci. U.S.A. 2009, 106, 6048-6053.

(13) Penn, R. L.; Banfield, J. F. Geochim. Cosmochim. Acta 1999, 63, 1549- 1557.

(14) Banfield, J. F.; Welch, S. A.; Zhang, H. Z.; Ebert, T. T.; Penn, R. L. Science 2000, 289, 751-754.

(15) Co“lfen, H.; Antonietti, M. Mesocrystals and Nonclassical Crystallization; John Wiley \& Sons: Chichester, U.K., 2008.

(16) Politi, Y.; Metzler, R. A.; Abrecht, M.; Gilbert, B.; Wilt, F. H.; Sagi, I.; Addadi, L.; Weiner, S.; Gilbert, P. U. P. A. Proc. Natl. Acad. Sci.

U.S.A. 2008, 105, 17362-17366.

(17) Aizenberg, J.; Muller, D. A.; Grazul, J. L.; Hamann, D. R. Science 2003, 299, 1205-1208.

(18) Aizenberg, J.; Albeck, S.; Weiner, S.; Addadi, L. J. Cryst. Growth 1994, 142, 156-164.

(19) Metzler, R. A.; Abrecht, M.; Olabisi, R. M.; Ariosa, D.; Johnson, C. J.; Frazer, B. H.; Coppersmith, S. N.; Gilbert, P. U. P. A. Phys. ReV. Lett. 2007, 98, 268102.

(20) Metzler, R. A.; Zhou, D.; Abrecht, M.; Chiou, J.-W.; Guo, J.; Ariosa, D.; Coppersmith, S. N.; Gilbert, P. U. P. A. Phys. ReV.B 2008, 77, 064110.

(21) Gilbert, P. U. P. A.; Metzler, R. A.; Zhou, D.; Scholl, A.; Doran, A.; Young, A.; Kunz, M.; Tamura,

N.; Coppersmith, S. N. J. Am. Chem. Soc. 2008, 130, 17519-17527.

(22) Ma“rkel, K. Zoomorphol. 1978, 89, 125-144.

(23) Ma“rkel, K. Zoomorphol. 1979, 94, 1-32.

(24) Birenheide, R.; Motokawa, T. Biol. Bull. 1996, 190, 218-230.

(25) Ma“rkel, K. Annot. Zool. Jpn. 1970, 43, 188-199.

(26) Khachaturyan, A. G. Theory of Structural Transformation in Solids; Wiley: New York, 1983.

(27) Lee, J. R. I.; Han, T. Y.-J.; Willey, T. M.; Wang, D.; Meulenberg,

R. W.; Nilsson, J.; Dove, P. M.; Terminello, L. J.; van Buuren, T.; De Yoreo, J. J. J. Am. Chem. Soc. 2007, 129, 10370-10381.

(28) Park, R. J.; Meldrum, F. C. AdV. Mater. 2002, 14, 1167-1169.

(29) Pichon, B. P.; Bomans, P. H. H.; Frederik, P. M.; Sommerdijk, N. A. J. M. J. Am. Chem. Soc. 2008 , 130, 4034-4040.

(30) Beniash, E.; Aizenberg, J.; Addadi, L.; Weiner, S. Proc. R. Soc. London, Ser. B 1997, 264, 461-465.

(31) Politi, Y.; Arad, T.; Klein, E.; Weiner, S.; Addadi, L. Science 2004, 306, 1161-1164.

(32) Nassif, N.; Pinna, N.; Gehrke, N.; Antonietti, M.; Jager, C.; Co“lfen,

H. Proc. Natl. Acad. Sci. U.S.A. 2005, 102, 12653-12655.

(33) Weiss, I. M.; Tuross, N.; Addadi, L.; Weiner, S. J. Exp. Zool. 2002, 293, 478-491.

(34) Beniash, E.; Metzler, R. A.; Lam, R. S. K.; Gilbert, P. U. P. A. J. Struct. Biol. 2009, 166, $133-143$.

(35) Mahamid, J.; Sharir, A.; Addadi, L.; Weiner, S. Proc. Natl. Acad. Sci. U.S.A. 2008, 105, 12748- 
12753.

(36) Addadi, L.; Raz, S.; Weiner, S. AdV. Mater. 2003, 15, 959-970.
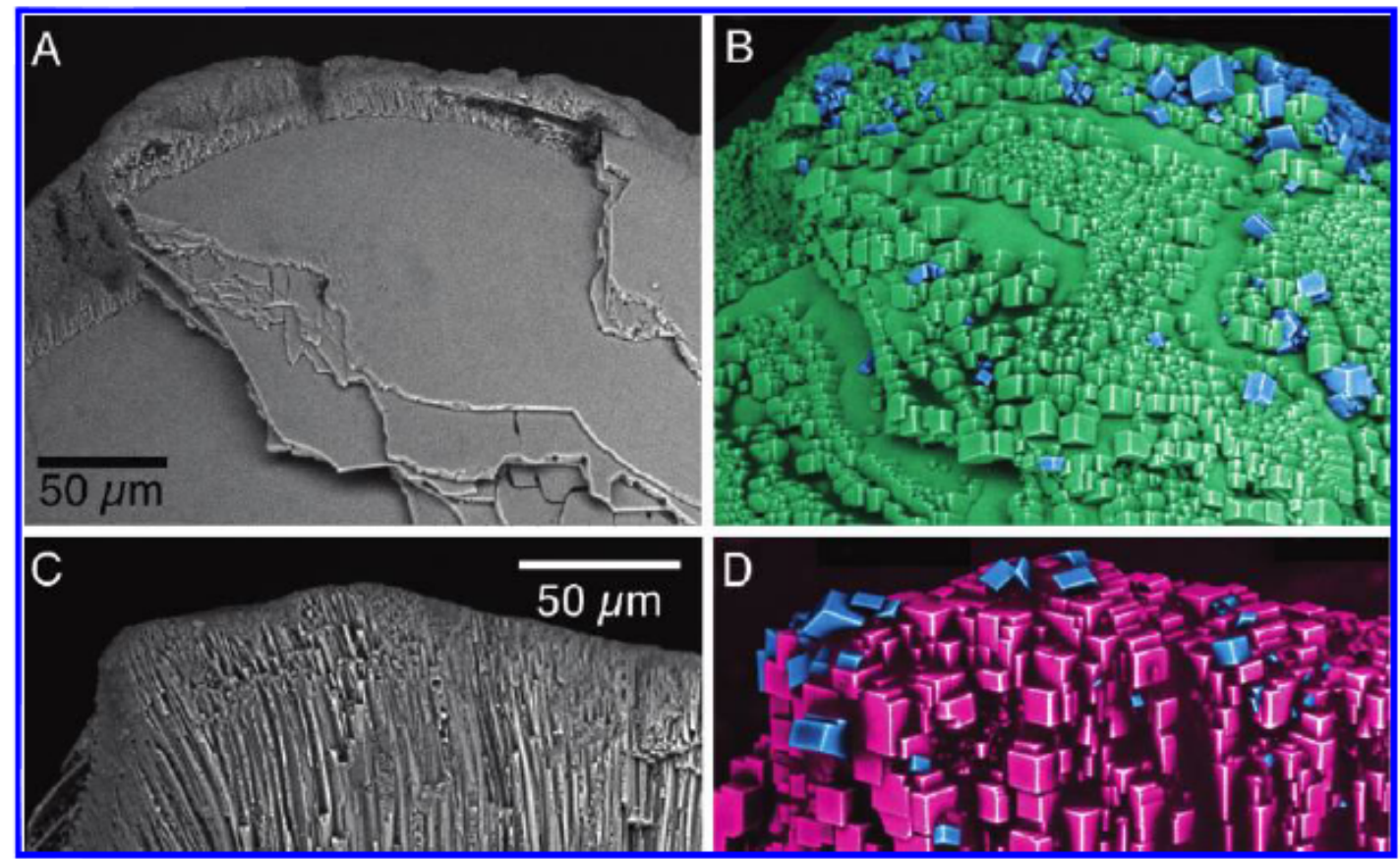

Figure 1. SEM micrographs of the tooth tip, before and after calcite crystal overgrowth. $(\mathrm{A}, \mathrm{C})$ The tip seen from the convex abaxial side and from the concave adaxial side, respectively. The convex side exhibits the surfaces of several plates, while the concave side shows the fibers and the polycrystalline matrix between them. (B) The region in (A), with overgrown and co-oriented calcite rhombohedra colored in green, and mis-oriented rhombohedra colored in blue. Mis-oriented rhombohedra appear at the edges of the plates, which the sea urchin broke while grinding. Broken-off tooth fragments provide misoriented nucleation sites for calcite overgrowth. The precise alignment of the overgrown rhombohedra in (B) shows that despite the curvature of the plates in both longitudinal and transverse directions, each plate is a single crystal, and all plates have the same orientation. (D) The same region as in (C), with overgrown and co-oriented rhombohedra colored in magenta, and the mis-oriented ones in blue. See Figure S1 for low-magnification SEM micrographs of these same regions. 


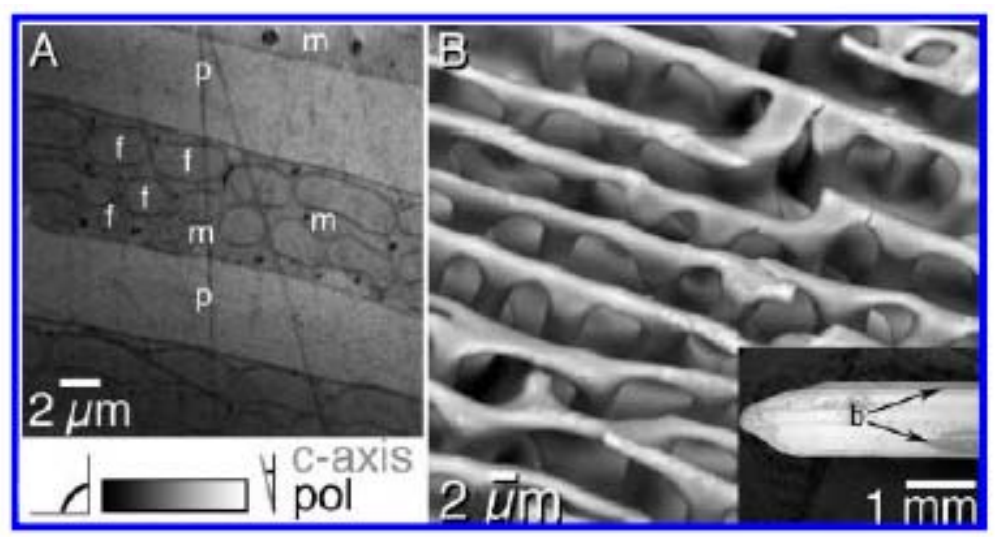

Figure 2. Details of the S. purpuratus tooth components. (A) This PIC map, acquired with X-PEEM on a tooth cross-section, shows the outlines of 2 plates (p), approximately 30 fibers (f), and the polycrystalline matrix (m) cementing plates and fibers together. The grayscale indicator shows qualitatively that gray levels correspond to different angles between the linear polarization vector (dark vertical line) and the $c$-axis (gray line). Notice the lighter gray level in the plates, indicating a different calcite $c$-axis orientation in the plates as compared to the fibers and matrix, which are darker. (B) SEM micrograph of the surface of an intact tooth, showing the pillar bridges on its outer surface. Each bridge has a "deck" running parallel to the tooth lateral edge, and many "piers" joining the bridge with the underlying plates. The inset shows a low-magnification SEM image of the tooth, with the tip on the left. The bridges (b) extend all the way from the forming end until approximately $3 \mathrm{~mm}$ from the tip (arrows), and then they are shed off and only the footprints of the bridge "piers" remain. See Figures S2-S5 for additional images of bridges on the teeth of $S$. purpuratus, $P$. lividus, and L variegatus urchins. 


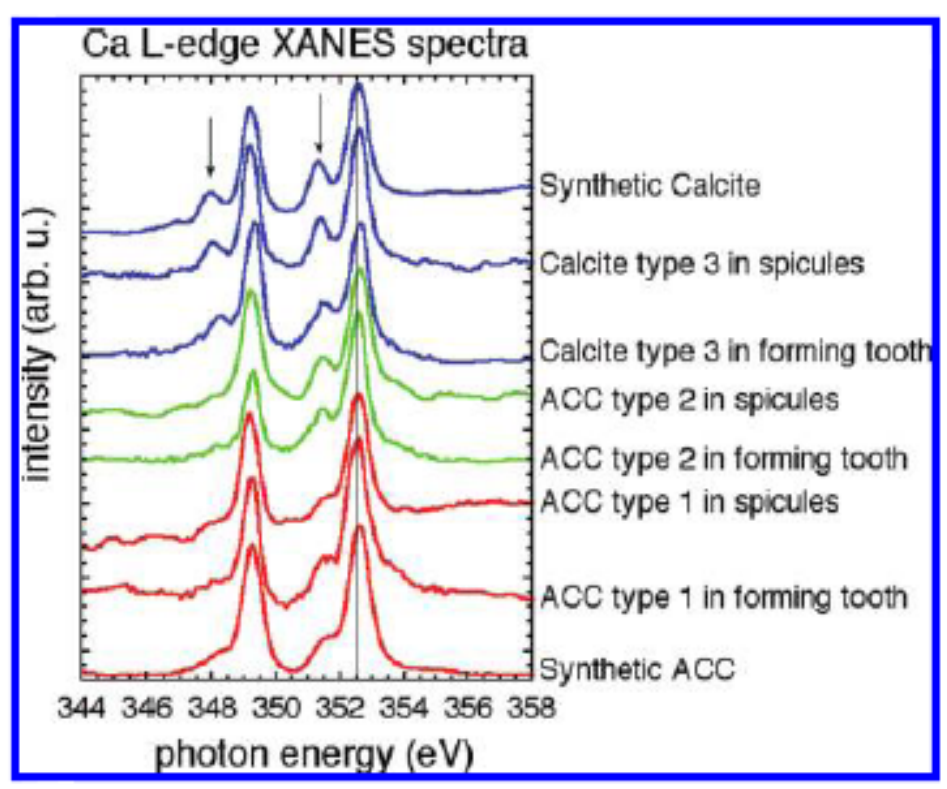

Figure 3. Calcium spectra from the forming end of the sea urchin tooth. The calcium L-edge spectra were acquired from the $20 \mathrm{~nm}$ pixels shown in Figure $\mathrm{S} 6$ and compared to those from ref 16 from sea urchin spicules, and to synthetic references. The spectra are distinguished by the relative intensities of the two crystal field peeks (arrows). Spicule spectra from type 1,2 , and $3 \mathrm{CaCO}_{3}$ are displayed in red, green, and blue. The spectra from $20 \mathrm{~nm}$ pixels in the forming tooth are in agreement with those from spicules, thus they are assigned to the same three types of $\mathrm{CaCO}_{3}$ and colored similarly. The first phase is hydrated ACC, termed type 1 ; its spectrum is characterized by the two crystal field peaks both having low intensity (low-low). The second phase, type 2, with one high intensity crystal field peak and one low intensity peak (low-high), is thought to be anhydrous ACC ${ }^{36}$ the third phase, type 3 , with two high intensity crystal field peaks (high-high), is crystalline calcite. No linear combination of high-high and low-low peaks could generate the low-high peaks observed in type 2 spectra; thus this is a distinct mineral phase separate from low-low type 1 and high-high type $3{ }^{16}$ Comparison with synthetic ACC and calcite reference standards confirms the interpretation that type 1 is hydrated ACC, and type 3 is crystalline calcite. No synthetic reference can be prepared for type 2, but because there is no volume or mass change upon crystallization, we conclude that this is likely anhydrous ACC. 


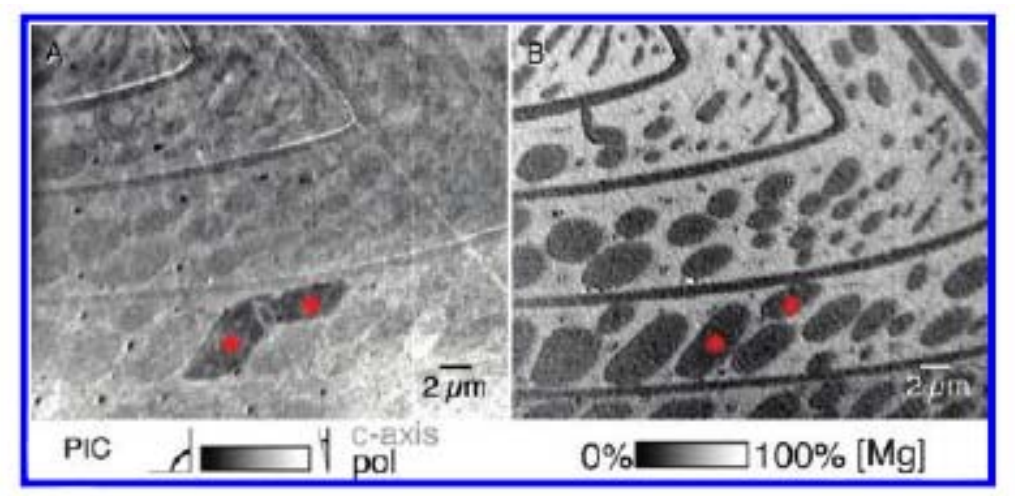

Figure 4. Two stray fibers reveal how co-orientation arises. X-PEEM results from a region of a cross-section at the mature end of the tooth, fully described in Figure S7. (A) PIC map showing two strongly mis-oriented fibers (red asterisks). (B) $\mathrm{Mg}$ distribution map from the same region as (A), showing well-defined and sharp elliptical fiber edges. Such strongly mis-aligned fibers are extremely rare in the sea urchin tooth, in which all other fibers are co-oriented with each other and with the polycrystalline matrix. Despite the sharp edges of the fibers in the Mg map, the PIC map shows that the polycrystalline matrix surrounding and between the two stray fibers is as mis-oriented as the fibers themselves. This indicates that the nanoparticles in the polycrystalline matrix get their orientation from the fibers. 


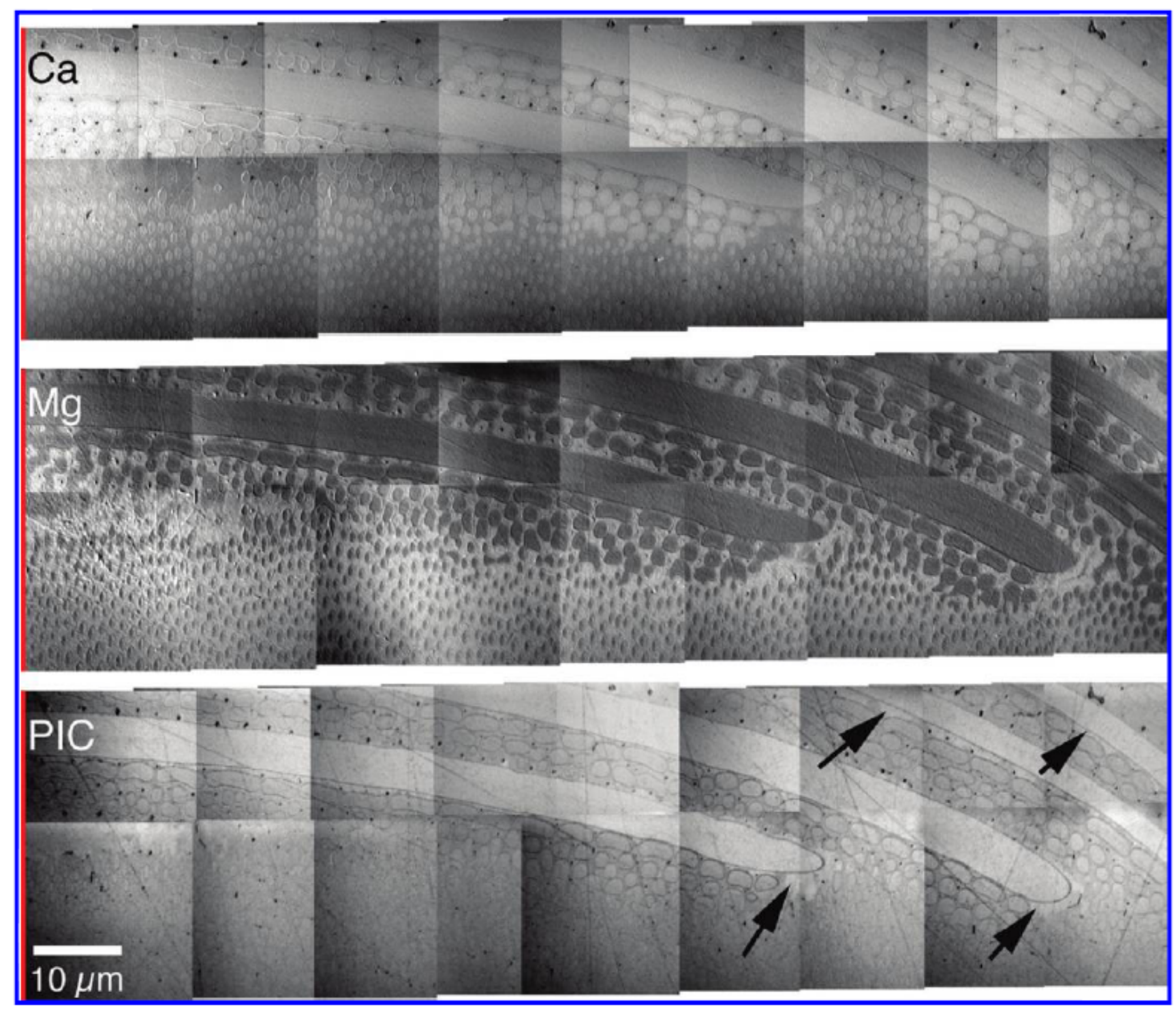

Figure 5. Elemental composition and crystal orientation in the sea urchin tooth stone part. $\mathrm{Ca}, \mathrm{Mg}$, and PIC maps acquired with PEEM on a sea urchin tooth cross-section near the center of the stone part, with portions of the first four plates visible. The entire tooth has mirror symmetry with respect to a vertical plane passing through its center, indicated by a red line in these images. The $\mathrm{Ca}$ and $\mathrm{Mg}$ distribution maps have inverted contrast, consistent with $\mathrm{Mg}$ replacing $\mathrm{Ca}$ in the matrix calcite. The PIC image shows lighter gray level in the primary plates, and a small portion of the polycrystalline matrix has the same orientation "spilling out" of the plates, where indicated by the arrows. (A gray level gradient across each map is noticeable. This is the result of uneven illumination across PEEM images at different energies and times. Each image-ratio map has a slightly different gradient, which was intentionally not corrected to avoid treating each map differently and somewhat arbitrarily.) 